\title{
Matrix Cones, Projection Representations, and Stable Set Polyhedra
}

\author{
L. LOVÁSZ AND A. SCHRIJVER
}

\begin{abstract}
It has been recognized recently that to represent a polyhedron as the projection of a higher-dimensional, but simpler, polyhedron is a powerful tool in polyhedral combinatorics. We develop a general method to construct higher-dimensional polyhedra (or, in some cases, convex sets) whose projection approximates the convex hull of $0-1$ valued solutions of a system of linear inequalities. An important feature of these approximations is that one can optimize any linear objective function over them in polynomial time.

In the special case of the vertex packing polytope, we obtain a sequence of systems of inequalities, such that already the first system includes clique, odd hole, odd antihole, wheel, and orthogonality constraints. In particular, for perfect (and many other) graphs, this first system gives the vertex packing polytope. For various classes of graphs, including $t$-perfect graphs, it follows that the stable set polytope is the projection of a polytope with a polynomial number of facets.
\end{abstract}

\section{Introduction}

One of the most important methods in combinatorial optimization is to represent each feasible solution of the problem by a $0-1$ vector (usually the incidence vector of the appropriate set), and then describe the convex hull $K$ of the solutions by a system of linear inequalities. In the nicest cases (e.g., in the case of the bipartite matching problem) we obtain a system that has polynomial size (measured in the natural "size" $n$ of the problem). In such a case, we can compute the maximum of any linear objective function in polynomial time by solving a linear program. In other cases, however, the convex hull of feasible solutions has exponentially many facets and so can only be described by a linear program of exponential size. For most of the polynomial time solvable combinatorial optimization problems, this exponentially large set of linear inequalities is still "nice" in one sense or another. We mention two possible notions of "niceness":

- Given an inequality in the system, there is a polynomial size certificate of

1980 Mathematics Subject Classification (1985 Revision). Primary 05C35, 90C10, 90C27. 
the fact that it is valid for $K$. If this is the case, the problem of determining whether a given vector lies in $K$ is in the complexity class co-NP.

- There is a polynomial time separation algorithm for the system; that is, given a vector, we can check in polynomial time whether it satisfies the system, and if not, we can find an inequality of the system that is violated. It follows then from general results on the ellipsoid method (see Grötschel, Lovász, and Schrijver [14]) that every linear objective function can be optimized over $K$ in polynomial time.

Many important theorems in combinatorial optimization provide such "nice" descriptions of polyhedra. Typically, to find such a system and to prove its correctness, one needs ad hoc methods depending on the combinatorial structure. However, one can mention two general ideas that can help in obtaining such linear descriptions:

- Gomory-Chvátal cuts. Let $P$ be a polytope with integral vertices. Assume that we have already found a system of linear inequalities valid for $P$ whose integral solutions are precisely the integral vectors in $P$. The solution set of this system is a polytope $K$ containing $P$, which will in general be larger than $P$. We can generate further linear inequalities valid for $P$ (but not necessarily for $K$ ) as follows. Given a linear inequality

$$
\sum_{i} a_{i} x_{i} \leq \alpha
$$

valid for $K$, where the $a_{i}$ are integers, the inequality

$$
\sum_{i} a_{i} x_{i} \leq\lfloor\alpha\rfloor
$$

is still valid for $P$ but may eliminate some part of $K$. Gomory [11] used a special version of this construction in his integer programming algorithm. If we take all inequalities obtainable this way, they define a polytope $K^{\prime}$ with $P \subseteq K^{\prime} \subset K$. Repeating this with $K^{\prime}$ in place of $K$ we obtain $K^{\prime \prime}$, etc. Chvátal [8] proved that in a finite number of steps we obtain the polytope $P$ itself.

Unfortunately, the number of steps needed may be very large; it depends not only on the dimension but also on the coefficients of the system we start with. Another trouble with this procedure is that there is no efficient way known to implement it algorithmically. In particular, even if we know how to optimize a linear objective function over $K$ in polynomial time (say, $K$ is given by an explicit, polynomial-size linear program), and $K^{\prime}=P$, we know of no general method to optimize a linear objective function over $P$ in polynomial time.

- Projection representation (new variables). This method has received much attention lately. The idea is that a projection of a polytope may have more facets than the polytope itself. This remark suggests that even if $P$ has exponentially many facets, we may be able to represent it as the projection of a polytope $Q$ in higher (but still polynomial) dimension, having only 
a polynomial number of facets. Among others, Ball, Liu, and Pulleyblank [3], Maculan [17], Balas and Pulleyblank [1], [2], Barahona and Mahjoub [5], Cameron and Edmonds [6] have provided nontrivial examples of such a representation. It is easy to see that such a representation can be used to optimize linear objective functions over $P$ in polynomial time. In the negative direction, Yannakakis [20] proved that the Travelling Salesman polytope and the Matching Polytope of complete graphs cannot be represented in this way, assuming that the natural symmetries of these polytopes are preserved by lifting. (No negative results seem to be known without this symmetry assumption.)

One way to view our results is that we provide a general procedure to create such liftings. The idea is to extend the method of Grötschel, Lovász, and Schrijver [12] for finding maximum stable sets in perfect graphs to general $0-1$ programs. We represent a feasible subset not by its incidence vector $v$ but by the matrix $v v^{\mathrm{T}}$. This squares the number of variables, but in return we obtain two new powerful ways to write down linear constraints. Projecting back to the "usual" space, we obtain a procedure somewhat similar to the Gomory-Chvátal procedure: it "cuts down" a convex set $K$ to a new convex set $K^{\prime}$ so that all $0-1$ solutions are preserved. In contrast to the Gomory-Chvátal cuts, however, any subroutine to optimize a linear objective function over $K$ can be used to optimize a linear objective function over $K^{\prime}$. Moreover, repeating the procedure at most $n$ times, we obtain the convex hull $P$ of $0-1$ vectors in $K$.

Our method is closely related to recent work of Sherali and Adams [19]. They introduce new variables for products of the original ones and characterize the convex hull, in this high-dimensional space, of vectors associated with $0-1$ solutions of the original problem. In this way they obtain a sequence of relaxations of the 0-1 optimization problem, the first of which is essentially the $N$ operator introduced in Section 1 below. (Further members of the two sequences of relaxations are different.) The method is also related to (but different from) the recent work of Pemantle, Propp, and Ullman [18] on the tensor powers of linear programs.

In Section 1, we describe the method in general, and give its basic properties. Section 2 contains applications to the vertex packing problem, one of the best studied combinatorial optimization problems. It will turn out that our method gives in one step almost all of the known classes of facets of the vertex packing polytope. It will follow in particular that if a graph has the property that its stable set polytope is described by the clique, odd hole, and odd antihole constraints, then its maximum stable set can be found in polynomial time.

In this paper, proofs are omitted or at most sketched. Full proofs, as well as a discussion of the extension of this method to higher tensor powers, will be published elsewhere. 
Acknowledgment. The first author is grateful to the Department of Combinatorics and Optimization of the University of Waterloo for its hospitality while this paper was written. Discussions with Mike Saks and Bill Pulleyblank on the topic of the paper were most stimulating.

\section{Matrix cuts}

In this section we describe a general construction for "lifting" a 0-1 programming problem in $n$ variables to $n^{2}$ variables, and then projecting it back to the $n$-space so that cuts, i.e., tighter inequalities still valid for all $0-1$ solutions, are introduced. It will be convenient to deal with homogeneous systems of inequalities, i.e., with convex cones rather than polytopes. Therefore we embed the $n$-dimensional space in $\mathbb{R}^{n+1}$ as the hyperplane $x_{0}=1$. (The 0 th variable will play a special role throughout.)

a. The construction of matrix cones and their projections. Let $K$ be a convex cone in $\mathbb{R}^{n+1}$. Let $K^{*}$ be its polar cone, i.e., the cone defined by

$$
K^{*}=\left\{u \in \mathbb{R}^{n+1}: u^{\mathrm{T}} x \geq 0 \text { for all } x \in K\right\} .
$$

We denote by $K^{0}$ the cone spanned by all $0-1$ vectors in $K$. Let $Q$ denote the cone spanned by all $0-1$ vectors $x \in \mathbb{R}^{n+1}$ with $x_{0}=1$. We are interested in determining $K^{o}$, and generally we may restrict ourselves to subcones of $Q$. We denote by $e_{i}$ the $i$ th unit vector, and set $f_{i}=e_{0}-e_{i}$. Note that the cone $Q^{*}$ is spanned by the vectors $e_{i}$ and $f_{i}$. For any $(n+1) \times(n+1)$ matrix $Y$, we denote by $\bar{Y}$ the vector composed of the diagonal entries of $Y$.

Let $K_{1} \subseteq Q$ and $K_{2} \subseteq Q$ be convex cones. We define the cone $M\left(K_{1}, K_{2}\right)$ $\subseteq \mathbb{R}^{(n+1) \times(n+1)}$ consisting of all $(n+1) \times(n+1)$ matrices $Y=\left(y_{i j}\right)$ satisfying (i), (ii), and (iii) below (for motivation, the reader may think of $Y$ as a matrix of the form $x x^{\mathrm{T}}$, where $x$ is a $0-1$ vector in $K_{1} \cap K_{2}$ ).

(i) $Y$ is symmetric;

(ii) $\bar{Y}=Y e_{0}$, i.e., $y_{i i}=y_{0 i}$ for all $1 \leq i \leq n$;

(iii) $u^{\mathrm{T}} Y v \geq 0$ holds for every $u \in K_{1}^{*}$ and $v \in K_{2}^{*}$. Note that (iii) can be rewritten as

(iii') $Y K_{2}^{*} \subseteq K_{1}$.

We shall also consider a slightly more complicated cone $M_{+}\left(K_{1}, K_{2}\right)$, consisting of matrices $Y$ satisfying the following condition in addition to (i), (ii), and (iii):

(iv) $Y$ is positive semidefinite.

From the assumption that $K_{1}$ and $K_{2}$ are contained in $Q$ it follows that every $Y=\left(y_{i j}\right) \in M\left(K_{1}, K_{2}\right)$ satisfies $y_{i j} \geq 0, y_{i j} \leq y_{i i}=y_{0 i} \leq y_{00}$ and $y_{i j} \geq y_{i i}+y_{j j}-y_{00}$.

These cones of matrices are defined by linear constraints and so their polars can also be expressed quite nicely. Let $U_{\text {psd }}$ denote the cone of positive semidefinite $(n+1) \times(n+1)$ matrices (which is self-dual in the space 
$U_{\text {sym }}$ of symmetric matrices), and $U_{\text {skew }}$ the linear space of skew symmetric $(n+1) \times(n+1)$ matrices (which is the orthogonal complement of $\left.U_{\text {sym }}\right)$. Let $U_{1}$ denote the linear space of $(n+1) \times(n+1)$ matrices $\left(w_{i j}\right)$, where $w_{0 j}=-w_{j j}$ for $1 \leq j \leq n, w_{00}=0$ and $w_{i j}=0$ if $i \neq 0$ and $i \neq j$. Note that $U_{1}$ is generated by the matrices $f_{i} e_{i}^{\mathrm{T}}(i=1, \ldots, n)$.

With this notation, we have by definition

$$
M\left(K_{1}, K_{2}\right)^{*}=U_{1}+U_{\text {skew }}+\operatorname{cone}\left\{u v^{\mathrm{T}}: u \in K_{1}^{*}, v \in K_{2}^{*}\right\},
$$

and

$$
M_{+}\left(K_{1}, K_{2}\right)^{*}=U_{1}+U_{\text {skew }}+U_{\text {psd }}+\operatorname{cone}\left\{u v^{\mathrm{T}}: u \in K_{1}^{*}, v \in K_{2}^{*}\right\} .
$$

Note that only the last term depends on the cones $K_{1}$ and $K_{2}$. In this term, it would be enough to let $u$ and $v$ run over extreme rays of $K_{1}^{*}$ and $K_{2}^{*}$, respectively. So if $K_{1}$ and $K_{2}$ are polyhedral, then so is $M\left(K_{1}, K_{2}\right)$, and the number of its facets is at most the product of the numbers of facets of $K_{1}$ and $K_{2}$.

Note that $U_{\text {psd }}$ and hence $M_{+}\left(K_{1}, K_{2}\right)$ will in general be nonpolyhedral.

We project down these cones from the $(n+1) \times(n+1)$-dimensional space to the $(n+1)$-dimensional space by letting

$$
N\left(K_{1}, K_{2}\right)=\left\{Y e_{0}: Y \in M\left(K_{1}, K_{2}\right)\right\}=\left\{\bar{Y}: Y \in M\left(K_{1}, K_{2}\right)\right\}
$$

and

$$
N_{+}\left(K_{1}, K_{2}\right)=\left\{Y e_{0}: Y \in M_{+}\left(K_{1}, K_{2}\right)\right\}=\left\{\bar{Y}: Y \in M_{+}\left(K_{1}, K_{2}\right)\right\} .
$$

Clearly $M\left(K_{1}, K_{2}\right)=M\left(K_{2}, K_{1}\right)$ and so $N\left(K_{1}, K_{2}\right)=N\left(K_{2}, K_{1}\right)$ (and similarly for the " + " subscripts).

If $A \in \mathbb{R}^{(n+1) \times(n+1)}$ is a linear transformation mapping the cone $Q$ onto itself, clearly $M\left(A K_{1}, A K_{2}\right)=A M\left(K_{1}, K_{2}\right) A^{\mathrm{T}}$. If $n \geq 2$ then from $A Q=$ $Q$ it also follows that $A^{\mathrm{T}} e_{0}$ is parallel to $e_{0}$, and hence $N\left(A K_{1}, A K_{2}\right)=$ $A N\left(K_{1}, K_{2}\right)$. In particular, we can "flip" coordinates replacing $x_{i}$ by $x_{0}-x_{i}$ for some $i \neq 0$.

If $K_{1}$ and $K_{2}$ are polyhedral cones then $M\left(K_{1}, K_{2}\right)$ and $N\left(K_{1}, K_{2}\right)$ are as well. The cones $M_{+}\left(K_{1}, K_{2}\right)$ and $N_{+}\left(K_{1}, K_{2}\right)$ are also convex (but in general not polyhedral), since (iv) is equivalent to an infinite number of linear inequalities.

From the motivation given for the matrices $Y$ it is easy to deduce

$$
\text { 1.1. Lemma. }\left(K_{1} \cap K_{2}\right)^{o} \subseteq N_{+}\left(K_{1}, K_{2}\right) \subseteq N\left(K_{1}, K_{2}\right) \subseteq K_{1} \cap K_{2} \text {. }
$$

We will see that in general $N\left(K_{1}, K_{2}\right)$ will be much smaller than $K_{1} \cap K_{2}$.

The reason why we consider two convex cones instead of one is technical. We shall only need two special choices: either $K_{1}=K_{2}=K$ or $K_{1}=K$, $K_{2}=Q$. In fact, it is this second choice which behaves algorithmically better and which we study most of the time. To simplify notation, we set 
$N(K)=N(K, Q)$ and $M(K)=M(K, Q)$. In this case, $K_{2}^{*}=Q^{*}$ is generated by the vectors $e_{i}$ and $f_{i}$, and hence (iii') has the following convenient form:

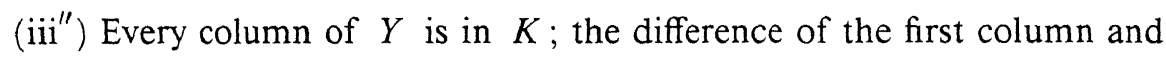
any other column is in $K$.

b. Properties of the cut operators. We give a lemma that yields a more explicit representation of constraints valid for $N(K)$ and $N_{+}(K)$. The proof is rather straightforward but a little lengthy.

1.2. Lemma. Let $K \subseteq Q$ be a convex cone in $\mathbb{R}^{n+1}$ and $a \in \mathbb{R}^{n+1}$.

(a) $w \in N(K)^{*}$ if and only if there exist vectors $a_{1}, \ldots, a_{n} \in K^{*}$, a real number $\lambda$, and a skew symmetric matrix $A$ such that $a_{i}+\lambda e_{i}+A e_{i} \in K^{*}$ for $i=1, \ldots, n$, and $w=\sum_{i=1}^{n} a_{i}+A \mathbb{1}$ (where $\mathbb{1}$ denotes the all-1 vector).

(b) $w \in N_{+}(K)^{*}$ if and only if there exist vectors $a_{1}, \ldots, a_{n} \in K^{*}$, a real number $\lambda$, a positive semidefinite symmetric matrix $B$, and a skew symmetric matrix $A$ such that $a_{i}+\lambda e_{i}+A e_{i}+B e_{i} \in K^{*}$ for $i=1, \ldots, n$, and $w=$ $\sum_{i=1}^{n} a_{i}+A \mathbb{1}+B \mathbb{1}$.

Our next lemma gives a geometric property of $N(K)$, which is easier to apply than the algebraic properties discussed before. Its proof follows quite easily from (iii"). Let

$$
H_{i}=\left\{x \in \mathbb{R}^{n+1}: x_{i}=0\right\} \text { and } G_{i}=\left\{x \in \mathbb{R}^{n+1}: x_{i}=x_{0}\right\} .
$$

Clearly $H_{i}$ and $G_{i}$ are hyperplanes supporting $Q$ at a facet, and all facets of $Q$ are determined this way.

1.3. Lemma. For every $1 \leq i \leq n, N(K) \subseteq\left(K \cap H_{i}\right)+\left(K \cap G_{i}\right)$.

Let us point out the following consequence of this lemma: if $K \cap G_{i}=\{0\}$ then $N(K) \subseteq H_{i}$. If, in particular, $K$ meets both opposite facets of $Q$ only in the 0 vector, then $N(K)=\{0\}$. This may be viewed as a very degenerate case of Gomory-Chvátal cuts (see below for more on the connection with Gomory-Chvátal cuts).

One could define a purely geometric cutting procedure based on this lemma: for each cone $K \subseteq Q$, consider the cone

$$
N_{0}(K)=\bigcap_{i}\left(\left(K \cap G_{i}\right)+\left(K \cap H_{i}\right)\right) .
$$

This cone is similar to $N(K)$ but in general bigger. We remark that this cone could also be obtained from a rather natural matrix cone by projection: this arises by imposing (ii), (iii), and the following restricted form of (i): $y_{0 i}=y_{i 0}$ for $i=1, \ldots, n$.

Figure 1 shows the intersection of three cones in $\mathbb{R}^{3}$ with the hyperplane $x_{3}=1$ : the cones $K, N(K)$, and $N(N(K))$, and the constraints implied by Lemma 1.3. We see that Lemma 1.3 gets close to $N(K)$ but does not determine it exactly. 


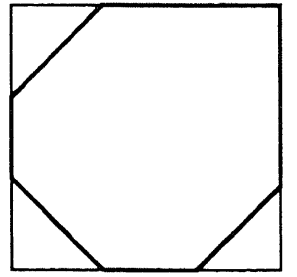

$K$

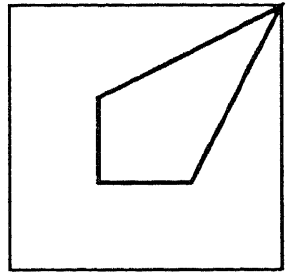

$N(K)$

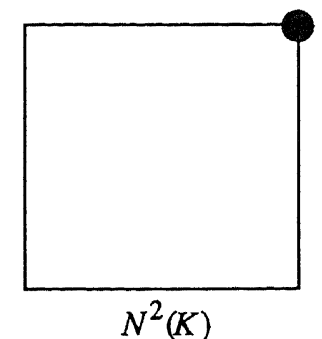

$N^{2}(K)$

FIGURE 1

We remark that $N\left(K \cap H_{i}\right)=N(K) \cap H_{i}$ for $i=1, \ldots, n$; it should be noted that $N\left(K \cap H_{i}\right)$ does not depend on whether it is computed as a cone in $\mathbb{R}^{n+1}$ or in $H_{i}$.

We can get better approximation of $K^{0}$ by iterating the operator $N$. Define $N^{t}(K)$ recursively by $N^{0}(K)=K$ and $N^{t}(K)=N\left(N^{t-1}(K)\right)$ for $t>0$.

\subsection{Theorem. $N^{n}(K)=K^{0}$.}

Next we show that if we use positive semidefiniteness, i.e., we consider $N_{+}(K)$, then an analogue of Lemma 1.3 can be obtained, which is more complicated but important in the applications to combinatorial polyhedra. Since the way positive semidefiniteness enters is not so obvious, we give the proof.

1.5. LemMA. Let $K \subseteq Q$ be a convex cone and let $a \in \mathbb{R}^{n+1}$ be a vector such that $a_{i} \leq 0$ for $i=1, \ldots, n$ and $a_{0} \geq 0$. Assume that $a^{\mathrm{T}} x \geq 0$ is valid for $K \cap G_{i}$ for all $i$ such that $a_{i}<0$. Then $a^{\mathrm{T}} x \geq 0$ is valid for $N_{+}(K)$.

(The condition that $a_{0} \geq 0$ excludes only trivial cases. The condition that $a_{i} \leq 0$ is a normalization, which can be achieved by flipping coordinates.)

Proof. First, assume that $a_{0}=0$. Consider a subscript $i$ such that $a_{i}<0$. (If no such $i$ exists, we have nothing to prove.) Then for every $x \in G_{i} \backslash\{0\}$, we have $a^{\mathrm{T}} x \leq a_{i} x_{i}<0$, and so $x \notin K$. Hence $K \cap G_{i}=\{0\}$ and so by Lemma 1.3, $N_{+}(K) \subseteq N(K) \subseteq K \cap H_{i}$. As this is true for all $i$ with $a_{i}<0$, we know that $a^{\mathrm{T}} x=0$ for all $x \in N_{+}(K)$.

Second, assume that $a_{0}>0$. Let $x \in N_{+}(K)$ and let $Y \in M_{+}(K)$ be a matrix with $Y e_{0}=x$. For any $1 \leq i \leq n$, the vector $Y e_{i}$ is in $K$ by $\left(\mathrm{iii}^{\prime \prime}\right)$ and in $G_{i}$ by (ii); so by the assumption on $a, a^{\mathrm{T}} Y e_{i} \geq 0$ whenever $a_{i}<0$. Hence $a^{\mathrm{T}} Y\left(a_{0} e_{0}-a\right)=a^{\mathrm{T}} Y\left(-a_{1} e_{1}-\cdots-a_{n} e_{n}\right) \geq 0$ (since those terms with $a_{i}=0$ do not contribute to the sum anyway), and hence $a^{\mathrm{T}} Y\left(a_{0} e_{0}\right) \geq$ $a^{\mathrm{T}} Y a \geq 0$ by positive semidefiniteness. Thus $a^{\mathrm{T}} Y e_{0}=a^{\mathrm{T}} x \geq 0$.

c. Algorithmic aspects. Next we turn to some algorithmic aspects of these constructions. We have to start by sketching the framework we are using; for a detailed discussion, see Grötschel, Lovász, and Schrijver [14]. 
Let $K$ be a convex cone. A strong separation oracle for the cone $K$ is a subroutine that, given a vector $x \in \mathbb{Q}^{n+1}$, either returns that $x \in K$ or returns a vector $w \in K^{*}$ such that $x^{\mathrm{T}} w<0$. A weak separation oracle is a version of this that allows for numerical errors: its input is a vector $x \in \mathbb{Q}^{n}$ and a rational number $\varepsilon>0$, and it either returns the assertion that the euclidean distance of $x$ from $K$ is at most $\varepsilon$, or returns a vector $w$ such that $|w| \geq 1, w^{\mathrm{T}} x \leq \varepsilon$ and the euclidean distance of $w$ from $K^{*}$ is at most $\varepsilon$. If the cone $K$ is spanned by $0-1$ vectors then we can strengthen a weak separation oracle to a strong one in polynomial time.

Let us also recall the following consequence of the ellipsoid method: Given a weak separation oracle for a convex body, together with some technical information (say, the knowledge of a ball contained in the body and of another one containing the body), we can optimize any linear objective function over the body in polynomial time (again, allowing an arbitrarily small error). If we have a weak separation oracle for a cone $K$ then we can consider its intersection with the half-space $x_{0} \leq 1$; using the above result, we can solve various important algorithmic questions concerning $K$ in polynomial time. We mention here the weak separation problem for the polar cone $K^{*}$.

The next theorem follows by general results on the ellipsoid method from the definition of $N$ and $N_{+}$, and from the fact that positive semidefiniteness of a matrix can be checked in polynomial time.

1.6. ThEOREM. Suppose that we have a weak separation oracle for a convex cone $K \subseteq Q$. Then the weak separation problem for $N(K)$ as well as for $N_{+}(K)$ can be solved in polynomial time.

d. Stronger cut operators. We could use stronger versions of this procedure to get convex sets smaller than $N(K)$.

One possibility is to consider $N(K, K)$ instead of $N(K)=N(K, Q)$. It is clear that $N(K, K) \subseteq N(K)$. Trivially, Theorem 1.4 and Lemma 1.3 remain valid if we replace $N(K)$ by $N(K, K)$. Unfortunately, it is not clear whether Theorem 1.6 also remains valid. The problem is that now we have to check whether $Y K^{*} \subseteq K$ and unfortunately $K^{*}$ may have exponentially many, or even infinitely many, extreme rays. If $K$ is given by a system of linear inequalities then this is not a problem. So in this case we could consider the sequence $N(K, K), N(N(K, K), K)$, etc. This shrinks down faster to $K^{o}$ than $N^{t}(K)$, as we shall see in the next section.

The following strengthening of the projection step in the construction seems quite interesting. For $v \in \mathbb{R}^{n+1}$, let $M(K) v=\{Y v: Y \in M(K)\}$. So $N(K)=M(K) e_{0}$. Now define

$$
\widehat{N}(K)=\bigcap_{r \in \text { int }\left(Q^{-}\right)} M(K) v
$$


Note that the intersection can be written in the form

$$
\widehat{N}(K)=\bigcap_{u \in Q^{*}} M(K)\left(e_{0}+u\right) .
$$

It is easy to see that

$$
K^{o} \subseteq \widehat{N}(K) \subseteq N(K)
$$

The following lemma, whose proof is again straightforward, gives a different characterization of $\widehat{N}(K)$ :

1.7. Lemma. $x \in \widehat{N}(K)$ if and only if for every $w \in \mathbb{R}^{n+1}$ and every $u \in Q^{*}$ such that $\left(e_{0}+u\right) w^{\mathrm{T}} \in M(K)^{*}$, we have $w^{\mathrm{T}} x \geq 0$.

In other words, $\widehat{N}(K)^{*}$ is generated by those vectors $w$ for which there exists a $v \in \operatorname{int}\left(Q^{*}\right)$ such that $v w^{\mathrm{T}} \in M(K)^{*}$.

The cone $\widehat{N}(K)$ satisfies important constraints that the cones $N(K)$ and $N_{+}(K)$ do not. Let $b \in \mathbb{R}^{n+1}$, and define $F_{b}=\left\{x \in \mathbb{R}^{n+1}: b^{\mathrm{T}} x \geq 0\right\}$. The next lemma shows a connection between $N$ and $\widehat{N}$.

1.8. Lemma. Assume that $N\left(K \cap F_{b}\right)=\{0\}$. Then $-b \in \widehat{N}(K)^{*}$.

We can use this lemma to derive a geometric condition on $\widehat{N}(K)$ similar to Lemma 1.5.

1.9. Lemma. Let $K \subseteq Q$ be a convex cone and assume that $e_{0} \notin K$. Then

$$
\widehat{N}(K) \subseteq\left(K \cap G_{1}\right)+\cdots+\left(K \cap G_{n}\right) .
$$

In other words, if $a^{\mathrm{T}} x \geq 0$ is valid for all of the faces $K \cap G_{i}$ then it is also valid for $\widehat{N}(K)$.

Applying this lemma to the cone in Figure 1, we can see that we obtain $K^{o}$ in a single step. The next corollary of Lemma 1.9 implies that at least some of the Gomory-Chvátal cuts for $K$ are satisfied by $\widehat{N}(K)$.

1.10. Corollary. Let $1 \leq k \leq n$ and assume that $\sum_{i=1}^{k} x_{i}>0$ holds for every $x \in K$. Then $\sum_{i=1}^{k} x_{i} \geq x_{0}$ holds for every $x \in \widehat{N}(K)$.

The proof consists of applying Lemma 1.9 to the projection of $K$ on the first $k+1$ coordinates.

Unfortunately, we do not know if Theorem 1.6 remains valid for $\widehat{N}(K)$. Of course, the same type of projection can be defined starting with $M_{+}(K)$ or with $M(K, K)$ instead of $M(K)$, and properties analogous to those in Lemmas $1.8-1.9$ can be derived.

\section{Stable set polyhedra}

We apply the results in the previous section to the stable set problem. To this end, we first survey some known methods and results on the facets of stable set polytopes. 
a. Facets of stable set polyhedra and perfect graphs. Let $G=(V, E)$ be a graph with no isolated nodes. Let $\alpha(G)$ denote the maximum size of any stable set of nodes in $G$. For each $A \subseteq V$, let $\chi^{A} \in \mathbb{R}^{V}$ denote its incidence vector. The stable set polytope of $G$ is defined as

$$
\operatorname{STAB}(G)=\operatorname{conv}\left\{\chi^{A}: A \text { is stable }\right\} \text {. }
$$

So the vertices of $\operatorname{STAB}(G)$ are just the $0-1$ solutions of the system of linear inequalities:

$$
x_{i} \geq 0 \text { for each } i \in V
$$

and

$$
x_{i}+x_{j} \leq 1 \text { for each } i j \in E .
$$

In general, $\operatorname{STAB}(G)$ is much smaller than the solution set of (1)-(2), which we denote by $\operatorname{FRAC}(G)$ ("fractional stable sets"). In fact, they are equal if and only if the graph is bipartite. The polytope $\operatorname{FRAC}(G)$ has many nice properties; what we will need is that its vertices are half-integral vectors.

There are several classes of inequalities that are satisfied by $\operatorname{STAB}(G)$ but not necessarily by $\operatorname{FRAC}(G)$. Let us mention some of the most important classes. The clique constraints strengthen the class (2): for each clique $B$, we have

$$
\sum_{i \in B} x_{i} \leq 1 .
$$

Graphs for which (1) and (3) are sufficient to describe $\operatorname{STAB}(G)$ are called perfect. It was shown by Grötschel, Lovász, and Schrijver [12] that the weighted stable set problem can be solved in polynomial time for these graphs.

The odd hole constraints express the nonbipartiteness of the graph: if $C$ induces a chordless odd cycle in $G$ then

$$
\sum_{i \in C} x_{i} \leq \frac{1}{2}(|C|-1) .
$$

Of course, the same inequality holds if $C$ has chords; but in this case it easily follows from other odd hole constraints and edge constraints. Nevertheless, it will be convenient that if we apply an odd hole constraint, we do not have to check whether the circuit in question is chordless.

Graphs for which (1), (2), and (4) are sufficient to describe $\operatorname{STAB}(G)$ are called t-perfect. Graphs for which (1), (3), and (4) are sufficient are called h-perfect. It was shown by Grötschel, Lovász, and Schrijver [13] that the weighted stable set problem can be solved in polynomial time for $h$-perfect (and hence also for $t$-perfect) graphs.

The odd antihole constraints are defined by sets $D$ that induce a chordless odd cycle in the complement of $G$ :

$$
\sum_{i \in D} x_{i} \leq 2
$$


We shall see that the weighted stable set problem can be solved in polynomial time for all graphs for which (1)-(5) are enough to describe $\operatorname{STAB}(G)$ (and for many more graphs).

All constraints (2)-(5) are special cases of the rank constraints: let $U \subseteq V$ induce a subgraph $G_{U}$; then

$$
\sum_{i \in U} x_{i} \leq \alpha\left(G_{U}\right)
$$

Of course, many of these constraints are inessential. To specify some that are essential, let us call a graph $G \alpha$-critical if it has no isolated nodes and $\alpha(G-e)>\alpha(G)$ for every edge $e$. Chvátal [9] showed that if $G$ is a connected $\alpha$-critical graph then the rank constraint

$$
\sum_{i \in V(G)} x_{i} \leq \alpha(G)
$$

defines a facet of $\operatorname{STAB}(G)$.

(Of course, in this generality rank constraints are ill-behaved: given any one of them, we have no polynomial time procedure to verify that it is indeed a rank constraint, since we have no polynomial time algorithm to compute the stability number of the graph on the right-hand side. For the special classes of rank constraints introduced above, however, it is easy to verify that a given inequality belongs to them.)

Finally, we remark that not all facets of the stable set polytope are determined by rank constraints. For example, let $U$ induce an odd wheel in $G$, with center $u_{0} \in U$. Then the constraint

$$
\sum_{i \in U^{\prime} \backslash\left\{u_{0}\right\}} x_{i}+\frac{|U|-2}{2} x_{u_{0}} \leq \frac{|U|-2}{2}
$$

is called a wheel constraint. If, e.g., $V(G)=U$, then the wheel constraint induces a facet of the stable set polytope.

Another class of nonrank constraints of a rather different character are orthogonality constraints, introduced by Grötschel, Lovász, and Schrijver [13]. Let us associate with each vertex $i \in V$, a vector $v_{i} \in \mathbb{R}^{n}$, so that $\left|v_{i}\right|=1$ and nonadjacent vertices correspond to orthogonal vectors. Let $c \in \mathbb{R}^{n}$ with $|c|=1$. Then

$$
\sum_{i \in V^{i}}\left(c^{\mathrm{T}} v_{i}\right)^{2} x_{i} \leq 1
$$

is valid for $\operatorname{STAB}(G)$. The solution set of these constraints (together with the nonnegativity constraints) is denoted by $\mathrm{TH}(G)$. It is easy to show that

$$
\operatorname{STAB}(G) \subseteq \operatorname{TH}(G) \subseteq \operatorname{FRAC}(G) .
$$

In fact, $\operatorname{TH}(G)$ satisfies all the clique constraints. Note that there are infinitely many orthogonality constraints for a given graph, and $\mathrm{TH}(G)$ is in general nonpolyhedral (it is polyhedral if and only if the graph is perfect). 
The advantage of $\operatorname{TH}(G)$ is that every linear objective function can be optimized over it in polynomial time. The algorithm involves convex optimization in the space of matrices, and was the main motivation for our studies in the previous section. We shall see that these techniques give substantially better approximations of $\operatorname{STAB}(G)$ over which one can still optimize in polynomial time.

b. The " $N$ " operator. To apply the results in the previous section, we homogenize the problem by introducing a new variable $x_{0}$ and consider $\operatorname{STAB}(G)$ as a subset of the hyperplane $H_{0}$ defined by $x_{0}=1$. We denote by $\operatorname{ST}(G)$ the cone spanned by the vectors $\left(\begin{array}{l}1 \\ x^{4}\end{array}\right) \in \mathbb{R}^{V \cup\{0\}}$, where $A$ is a stable set. We get $\operatorname{STAB}(G)$ by intersecting $\operatorname{ST}(G)$ with the hyperplane $x_{0}=1$. Similarly, let $\operatorname{FR}(G)$ denote the cone spanned by the vectors $\left(\begin{array}{l}1 \\ x\end{array}\right)$ where $x \in \operatorname{FRAC}(G)$. Then $\operatorname{FR}(G)$ is determined by the constraints

$$
x_{i} \geq 0 \text { for each } i \in V \text {, }
$$

and

$$
x_{i}+x_{j} \leq x_{0} \text { for each } i j \in E .
$$

Since it is often easier to work in the original $n$-dimensional space (without homogenization), we shall use the notation $N(\operatorname{FRAC}(G))=N(\operatorname{FR}(G)) \cap H_{0}$ and similarly for $N_{+}, \widehat{N}$, etc. We shall also abbreviate $N(\operatorname{FRAC}(G))$ by $N(G)$, etc. Since $\operatorname{FRAC}(G)$ is defined by an explicit linear program, one can solve the separation problem for it in polynomial time. We shall say briefly that the polytope is polynomial time separable. By Theorem 1.6, we obtain the following.

2.1. TheOREM. For each fixed $r \geq 0, N_{+}^{r}(G)$ as well as $N^{r}(G)$ are polynomial time separable.

It should be remarked that, in most cases, if we use $N^{r}(G)$ as a relaxation of $\operatorname{STAB}(G)$ then it does not really matter whether the separation subroutine returns hyperplanes separating the given $x \notin N^{r}(G)$ from $N^{r}(G)$ or only from $\operatorname{STAB}(G)$. Hence it is seldom relevant to have a separation subroutine for a given relaxation, say $N^{r}(G)$; one could use just as well a separation subroutine for any other convex body containing $\operatorname{STAB}(G)$ and contained in $N^{r}(G)$ (such as, e.g., $N_{+}^{r}(G)$ ). Hence the polynomial time separability of $N_{+}^{r}(G)$ is substantially deeper than the polynomial time separability of $N^{r}(G)$ (even though it does not imply it directly).

In the rest of this section we study the question of how much this theorem gives us: which graphs satisfy $N_{+}^{r}(G)=\operatorname{STAB}(G)$ for small values of $r$, and more generally, which of the known constraints are satisfied by $N(G), N_{+}(G)$, etc. With a little abuse of terminology, we shall not distinguish between the original and homogenized versions of clique, odd hole, etc. constraints. 
It is a useful observation that if $Y=\left(y_{i j}\right) \in M(\mathrm{FR}(G))$ then $y_{i j}=0$ whenever $i j \in E(G)$. In fact the constraint $x_{i}+x_{j} \leq 1$ must be satisfied by $Y e_{i}$, and so $y_{i i}+y_{j i} \leq y_{0 i}=y_{i i}$ by nonnegativity. This implies $y_{i j}=0$.

Let $a^{\mathrm{T}} x \leq b$ be any inequality valid for $\operatorname{STAB}(G)$. Let $W \subseteq V$ and let $a_{W} \in \mathbb{R}^{W}$ be the restriction of $a$ to $W$. For every $v \in V$, if $a^{\mathrm{T}} x \leq$ $b$ is valid for $\operatorname{STAB}(G)$ then $a_{V-v}^{\mathrm{T}} x \leq b$ is valid for $\operatorname{STAB}(G-v)$ and $a_{V-\Gamma(v)-v}^{\mathrm{T}} x \leq b-a_{v}$ is valid for $\operatorname{STAB}(G-\Gamma(v)-v$ ) (here $\Gamma(v)$ denotes the set of neighbors of the node $v$ ). Let us say that these inequalities arise from $a^{\mathrm{T}} x \leq b$ by the deletion and contraction of node $v$, respectively. Note that if $a^{\mathrm{T}} x \leq b$ is an inequality such that for some $v$, both the deletion and contraction of $v$ yield inequalities valid for the corresponding graphs, then $a^{\mathrm{T}} x \leq b$ is valid for $G$.

Let $K$ be any convex body such that $K$ contains $\operatorname{STAB}(G)$ and is contained in $\operatorname{FRAC}(G)$. Now Lemma 1.3 implies

2.2. LEMMA. If $a^{\mathrm{T}} x \leq b$ is an inequality valid for $\operatorname{STAB}(G)$ such that for some $v \in V$, both the deletion and contraction of $v$ give an inequality valid for $K$ then $a^{\mathrm{T}} x \leq b$ is valid for $N(G)$.

This lemma enables us to completely characterize the constraints obtained in one step (not using positive semidefiniteness):

2.3. THEOREM. The polytope $N(G)$ is exactly the solution set of the nonnegativity, edge, and odd hole constraints.

The fact that $N(G)$ satisfies these constraints follows easily from Lemma 2.2. The proof of the equality is much more difficult.

2.4. CoRollary. If $G$ is t-perfect then $\operatorname{STAB}(G)$ is the projection of a polytope whose number of facets is polynomial in $n$.

This corollary generalizes a result of Barahona and Mahjoub [5] that constructs such a projection representation for series-parallel graphs.

c. The repeated " $N$ " operator. Next we prove a theorem which describes a large class of inequalities valid for $N^{r}(G)$ for a given $r$. The result is not as complete as in the case $r=1$, but it does show that the number of constraints obtainable grows very quickly with $r$.

Let $a^{\mathrm{T}} x \leq b$ be any inequality valid for $\operatorname{STAB}(G)$. By Theorem 1.4, there exists an $r \geq 0$ such that $a^{\mathrm{T}} x \leq b$ is valid for $N^{r}(G)$. Let the $N$ index of the inequality be defined as the least $r$ for which this is true. We can define (and will study later) the $N_{+}$-index, $\widehat{N}$-index, etc. analogously. Note that in each version, the index of an inequality depends only on the subgraph induced by those nodes having a nonzero coefficient. In particular, if these nodes induce a bipartite graph then the inequality has $N$-index 0 . We can define the $N$-index of a graph as the largest $N$-index of the facets of $\operatorname{STAB}(G)$. The $N$-index of $G$ is 0 if and only if $G$ is bipartite; the 
$N$-index of $G$ is 1 if and only if $G$ is $t$-perfect. Lemma 2.2 implies (using the obvious fact that the $N$-index of an induced subgraph is never larger than the $N$-index of the whole graph):

2.5. Corollary. If for some node $v, G-v$ has $N$-index $k$ then $G$ has $N$-index at most $k+1$.

The following lemma about the iteration of the operator $N$ is useful in estimating the $N$-index of a constraint. The proof is easy by induction on $k$.

2.6. Lemma. $\frac{1}{k+2} \mathbb{1} \in N^{k}(G) \quad(k \geq 0)$.

From these two facts, we can derive some useful bounds on the $N$-index of a graph.

2.7. Corollary. Let $G$ be a graph with $n$ nodes and at least one edge. Assume that $G$ has stability number $\alpha(G)=\alpha$ and $N$-index $k$. Then

$$
\frac{n}{\alpha}-2 \leq k \leq n-\alpha-1 \text {. }
$$

It follows, in particular, that the $N$-index of a complete graph on $t$ nodes is $t-2$. The $N$-index of an odd hole is 1 , as an odd hole is a $t$-perfect graph. The $N$-index of an odd antihole with $2 k+1$ nodes is $k$; more generally, we have the following corollary.

2.8. Corollary. The $N$-index of a perfect graph $G$ is $\omega(G)-2$. The $N$-index of a critically imperfect graph $G$ is $\omega(G)-1$.

Next we study the index of a single inequality. Let $a^{\mathrm{T}} x \leq b$ be any constraint valid for $\operatorname{STAB}(G) \quad\left(a \in \mathbb{Z}_{+}^{V}, b \in \mathbb{Z}_{+}\right)$. Define the defect of this inequality as $2 \cdot \max \left\{a^{\mathrm{T}}-b: x \in \operatorname{FRAC}(G)\right\}$. The factor 2 in front guarantees that this is an integer. In the special case when we consider the constraint $\sum_{i} x_{i} \leq \alpha(G)$ for an $\alpha$-critical graph $G$, the defect is just the Gallai class number of the graph (see Lovász and Plummer [16] for a discussion of $\alpha$ critical graphs, in particular of the Gallai class number).

Given a constraint, its defect can be computed in polynomial time, since optimizing over $\operatorname{FRAC}(G)$ is an explicit linear program. The defect of a constraint is particularly easy to compute if the constraint defines a facet of $\operatorname{STAB}(G)$. This is shown by the following lemma, which states a property of facets of $\operatorname{STAB}(G)$ of independent interest.

2.9. Lemma. Let $\sum_{i} a_{i} x_{i} \leq b$ define a facet of $\operatorname{STAB}(G)$, different from those determined by the nonnegativity and edge constraints. Then every vector $v$ maximizing $a^{\mathrm{T}} x$ over $\operatorname{FRAC}(G)$ has $v_{i}=1 / 2$ whenever $a_{i}>0$. In particular,

and the defect of the inequality is $\sum_{i} a_{i}-2 b$.

$$
\max \left\{a^{\mathrm{T}} x: x \in \operatorname{FRAC}(G)\right\}=\frac{1}{2} \sum_{i} a_{i}
$$


Now we can state and prove our theorem that shows the connection between defect and the $N$-index.

2.10. THEOREM. Let $a^{\mathrm{T}} x \leq b$ be an inequality with integer coefficients valid for $\operatorname{STAB}(G)$ with defect $r$ and $N$-index $k$. Then

$$
\frac{r}{b} \leq k \leq r .
$$

If follows from our discussions that for an odd antihole constraint, the lower bound is tight. On the other hand, it is not difficult to check that for a rank constraint defined by an $\alpha$-critical subgraph that arises from $K_{p}$ by subdividing an edge by an even number of nodes, the upper bound is tight.

We want to mention that Ceria [7] proved that $N(\operatorname{FRAC}(G), \operatorname{FRAC}(G))$ also satisfies, among others, the $K_{4}$-constraints. We do not study the operator $K \mapsto N(K, K)$ here in detail, but a thorough comparison of its strength with $N$ and $N_{+}$would be very interesting.

d. The " $N_{+}$" operator. Now we turn to the study of the operator $N_{+}$for stable set polytopes. We do not have as general results as for the operator $N$, but we will be able to show that many constraints are satisfied even for very small $r$.

Lemma 2.3 implies

2.11. LEMMA. If $a^{\mathrm{T}} x \leq b$ is an inequality valid for $\mathrm{STAB}(G)$ such that for all $v \in V$ with a positive coefficient the contraction of $v$ gives an inequality with $N_{+}$-index at most $r$, then $a^{\mathrm{T}} x \leq b$ has $N_{+}$-index at most $r+1$.

The clique, odd hole, odd wheel, and odd antihole constraints have the property that contracting any node with positive coefficient we get an inequality in which the nodes with positive coefficients induce a bipartite subgraph. Hence

2.12. Corollary. Clique, odd hole, odd wheel, and odd antihole constraints have $N_{+}$-index 1 .

Hence all $h$-perfect (in particular all perfect and $t$-perfect) graphs have $N_{+}$-index at most 1 . We can also formulate the following recursive upper bound on the $N_{+}$-index of a graph.

2.13. Corollary. If $G-\Gamma(v)-v$ has $N_{+}$-index at most $r$ for every $v \in V$ then $G$ has $N_{+}$-index at most $r+1$.

Next, we consider the orthogonality constraints. To this end, consider the cone $M_{\mathrm{TH}}$ of $(V \cup\{0\}) \times(V \cup\{0\})$ matrices $Y=\left(y_{i j}\right)$ satisfying the following constraints:

(i) $Y$ is symmetric;

(ii) $y_{i i}=y_{i 0}$ for every $i \in V$; 
(iii') $y_{i j}=0$ for every $i j \in E$;

(iv) $Y$ is positive semidefinite.

As remarked, (iii') is a relaxation of (iii) in the definition of $M_{+}(\operatorname{FR}(G))$. Hence $M_{+}(\operatorname{FR}(G)) \subseteq M_{\mathrm{TH}}$.

2.14. Proposition. $\operatorname{TH}(G)=\left\{Y e_{0}: Y \in M_{\mathrm{TH}}, e_{0}^{\mathrm{T}} Y e_{0}=1\right\}$.

This representation of $\mathrm{TH}(G)$ is not a special case of the matrix cuts introduced in Section 1 (though clearly related). In a forthcoming paper we show that in fact, $\operatorname{TH}(G)$ is in a sense more fundamental than the relaxations of $\operatorname{STAB}(G)$ constructed in Section 1. Right now we can infer the following.

2.15. Corollary. Orthogonality constraints have $N_{+}$-index 1 .

We conclude with an upper bound on the $N_{+}$-index of a single inequality. Since $\alpha(G-\Gamma(v)-v)<\alpha(G)$, Lemma 2.3 gives by induction

2.16. Corollary. If $a^{\mathrm{T}} x \leq b$ is an inequality valid for $\operatorname{STAB}(G)$ such that the nodes with positive coefficients induce a graph with independence number $r$ then $a^{\mathrm{T}} x \leq b$ has $N_{+}$-index at most $r$. In particular, $a^{\mathrm{T}} x \leq b$ has index at most $b$.

Let us turn to the algorithmic aspects of these results. Theorem 2.1 implies

2.17. Corollary. The maximum weight stable set problem is polynomialtime solvable for graphs with bounded $N_{+}$-index.

Note that even for small values of $r$, quite a few graphs have $N_{+}$-index at most $r$. Collecting previous results, we obtain

2.18. Corollary. For any fixed $r \geq 0$, if $\operatorname{STAB}(G)$ can be defined by constraints $a^{\mathrm{T}} x \leq b$ such that either the defect of the constraint is at most $r$ or the support of a contains no stable set larger than $r$, then the maximum weight stable set problem is polynomial-time solvable for $G$.

\section{REFERENCES}

1. E. Balas and W. R. Pulleyblank, The perfect matchable subgraph polytope of a bipartite graph, Networks 13 (1983), 495-516.

2. The perfect matchable subgraph polytope of an arbitrary graph, Combinatorica (1987) (to appear).

3. M. O. Ball, W. Liu, and W. R. Pulleyblank, Two terminal Steiner tree polyhedra, Res. Rep. 87466-OR, Univ. Bonn, 1987.

4. F. Barahona, Reducing matching to polynomial size linear programming, Res. Rep. CORR 88-51, Univ. of Waterloo, 1988.

5. F. Barahona and A. R. Mahjoub, Compositions of graphs and polyhedra. II: Stable sets Res. Rep. 87464-OR, Univ. Bonn, 1987.

6. K. Cameron and J. Edmonds, Coflow polyhedra, preprint, Univ. of Waterloo, 1989.

7. S. Ceria, personal communication, 1989.

8. V. Chvátal, Edmonds polytopes and a hierarchy of combinatorial problems, Discrete Math. 4 (1973), 305-337.

9. $138-154$. 
10. J. Edmonds, Maximum matching and a polyhedron with 0-1 vertices, J. Res. Nat. Bur. Standards 69B (1965), 125-130.

11. R. E. Gomory, An algorithm for integer solutions to linear programs, in Recent advances in mathematical programming (ed. R. Graves and P. Wolfe), McGraw-Hill, 269-302, 1963.

12. M. Grötschel, L. Lovász, and A. Schrijver, The ellipsoid method and its consequences in combinatorial optimization, Combinatorica 1 (1981), 169-197.

13. Relaxations of vertex packing, J. Combin. Theory Ser. B 40 (1986), 330-343.

14. __ Geometric algorithms and combinatorial optimization, Springer, 1988.

15. W. Liu, Extended formulations and polyhedral projection, Thesis, Univ. of Waterloo, 1988.

16. L. Lovász and M. D. Plummer, Matching theory, Akadémiai Kiadó, Budapest, and Elsevier, Amsterdam, 1986.

17. N. Maculan, The Steiner problem in graphs, Ann. Discrete Math. 31 (1987), 185-222.

18. R. Pemantle, J. Propp, and D. Ullman, On tensor powers of integer programs, preprint (1989).

19. H. D. Sherali and W. P. Adams, A hierarchy of relaxations between the continuous and convex hull representations for zero-one programming problems, preprint (1988).

20. M. Yannakakis, Expressing combinatorial optimization problems by linear programs, Proc. 29th IEEE FOCS (1988), 223-228.

EÖtvös LORÁNd UNIVERsity, BUdAPEST AND PRINCETON UNIVERSITY

Mathematical Centre, Amsterdam 